\title{
HL-LHC industrialization and procurement. Lessons learnt
}

\section{Isabel Bejar Alonso ${ }^{1}$}

CERN

Address, Switzerland

E-mail: Isabel.BejarAlonsolcern.ch

The High-Luminosity LHC (LH-LHC) is a major upgrade of the Large Hadron Collider (LHC) that aims increasing the number of proton-proton collisions, boosting our chances of coming across new and rare physics phenomena. In order to do that, several new technologies will be introduced and heavy civil engineering work is required. Overall, more than $1.2 \mathrm{~km}$ of the current LHC ring will need to be replaced with new components.

The engineering concepts behind most part of these components come from our latest R\&D and only a few units will be required for each type. From one side producing them at CERN or in the laboratories collaborating in HL-LHC would be nearly impossible considering the production time frame. From the other side, the industry considers that the series are too short, the tolerances too tight and the development too risky to get interested. How to solve this situation?

The talk will give an overview of the sourcing strategy that was put in place for HL-LHC and the results obtained. Particular emphasis will be put on what we think were key success factors and could be useful for other construction projects facing the same challenges.

ICHEP2018,

5-11 July 2018

Seoul, Korea

\section{${ }^{1}$ Speaker}




\section{The HL-LHC project}

The High Luminosity LHC (HL-LHC) is a novel configuration of the Large Hadron Collider (LHC), aiming to achieve instantaneous luminosities a factor five or more above the nominal LHC design, thereby enabling the high luminosity experiments ATLAS and CMS to enlarge their data sample by one order of magnitude compared with the baseline programme.

The first step consisted of launching in 2011 a Design Study under the auspices of EC-FP7, which was instrumental in initiating a new global collaboration for the HL-LHC, matching the spirit of the worldwide user community of the LHC experiments. The HL-LHC was formally approved [1] by CERN Council in June 2016 becoming CERN's major construction project for the next decade. The aim is to have the first high luminosity beam in 2026 .

\section{The Organization, technology \& cost}

\subsection{Project structure}

The LHC Luminosity Upgrade was envisioned from the beginning as being an international project. Indeed, US laboratories started to work on it before the commissioning of the LHC and several European institution participated in Europeans programs such as CARE, SLH-PP and EuCARD to reinforce the design and R\&D work. The approval of the FP7 Design Study HiLumi LHC in 2011, allowed joining forces and creating a first structure based in Work Packages (WPs) and a governing structure with a collaboration board. Today 19 institutions are members of the collaboration board [2], 10 are collaboration partners and other 15 contribute with manpower and R\&D effort. The initial six WPs have grown to 19 where 14 are driven by new technologies and components and five take care of transversal tasks.

\subsection{Material and personnel Cost}

The cost of the collider upgrade, which will be realised within a constant CERN Budget, is estimated to be $950 \mathrm{M} \$$. The bottom-up evaluation of staff personnel requirements amounts to more than 1600 fulltime equivalent (FTE) years. Around $20 \%$ of the material and personnel value comes from in-kind contributions.

\section{The challenges}

\subsection{What type of challenges we will be facing}

There are several challenges linked to the development of a new accelerator. Some come from the technologies required and their technology maturity level. Others come from the industrialization of short series of components that are not COTS. Finally, there is the cost versus time factor. HL-LHC face all them. Equipment such as the $\mathrm{Nb}_{3} \mathrm{Sn}$ magnets, the crab cavities, the $\mathrm{MgB}_{2}$ links, or materials such as the MoGr for the collimators are completely new with a Technology Readiness Level close to 2 . For all them we require series that normally will be considered as the starting of the prototyping phase as we are talking about tens and not hundreds or thousands of identical components. None of them standard in the industry or part of what can be foreseen as a future product in their catalogue. 
Regarding timing, the timeline of the project is dictated by the fact that, at the beginning of the next decade, many critical components of the accelerator will reach the end of their lifetime due to radiation damage and will thus need to be replaced. Considering a standard development process this gives the project a maximum of 6 years from concept to a working prototype for objects as complex as a crab cavity for protons, an equipment never built before. HL-LHC is therefore crucial not only for the full exploitation of the LHC physics potential, but also to enable operation of the collider beyond 2026.

To these ones we have to add other challenges coming from the project structure. Large infrastructures are built with the contribution of different institutions that join efforts during the construction phase. Massive in-kind contributions are not easy to handle, as there is a delicate balance between the optimization of the infrastructure cost and the natural technological interest of each partner and their available funds. In-kind contributions require a special effort on interfaces and the definition of the acceptance criteria and reduce the possibility of last minute modifications to the design. It requires also distributed common tools to handle the interfaces and interlinked schedules.

The in-kind contributions is not the only challenge coming from the project structure. Projects require a massive injection of personnel in the structure of the hosting and collaborating labs. This new persons that will contribute to the project have short term contracts and will be up to 5 times the experienced permanent personnel. The existing personnel will have to assume the leading technological role, the training and guidance of the new personnel and all the administrative tasks linked to obtain the resources and stir the project. In the case of HL-LHC, less than $10 \%$ of the personnel will work for the full length of the project. Without a good administrative support unit and a project structure allowing the hiring of good experienced technologists and technicians at the right moment the project can face the deviation of the efforts of the leading persons to the administrative processes instead of letting them work on the success of the project.

\subsection{The work axis to face the challenges}

\subsubsection{Organization}

Working as a collaboration requires rules. Those willing to participate in HL-LHC contributing in terms of funds, expertise, equipment, materials and other in-kind contributions have to sign the HL-LHC Memorandum of Understanding (MoU). The MoU establishes the framework. It defines among others the governing bodies, the ownership, the intellectual property, the liability, the publication policy and the coordination among participants.

The integration of several research teams implies also a need of organizing the ownership of the results. For this reason, the project is cut in WPs where the full responsibility of the final delivery is on the hands of the WP Leader and the responsibility of the development of equipment and systems on the hands of WP Engineers. There is a clear definition and a Product Breakdown Structure, a Technical Design Report (TDR) [3] and of ownership. The technical WPs have the support of the transversal WPs that take care of ensuring that all the interfaces have been respected giving a holistic control of the project.

The project has a very strict review policy. All WPs are subject to Technical Design Reviews during the development stage and can only move to the production stage after a Production 
Readiness Review. During the fabrication, assembly and verification phase, there are Manufacturing Reviews that include suppliers audits [4].

\subsubsection{Make or buy process}

When facing the production of components with a Technology Readiness Level (TRL) [5] between 2 and 4 is crucial to analyse if is possible or not in only a few years to push it to TRL 9. The HL-LHC did a systematic analysis of each one of the components to be produced since the beginning of the project to identify what could be brought to TRL 9 and what not. This process called internally "Make or buy" identified also which components could be supplied by an inkind contribution. This early classification of the components did possible to make fact-based decisions such as launch competitive $R \& D$ for fast track $R \& D$ or transfer production $R \& D$ to the collaborations. The process was also the basis of the sourcing strategy as every year was explored which companies in the market could answer to the tenders of the components.

\subsubsection{Infrastructures}

Our clear vision of what could never be produced in industry gave us the need of internal production and testing infrastructures. What could not be "bought" had to be "made". Since 2014, there was a complete campaign to refurbish infrastructures and procurement of tooling to cope with in house production. This strategy was also a mitigation action in case of failure to produce in industry some of the components. During the construction of the LHC, this strategy had saved the project of numerous delays.

\subsubsection{Working with industry}

The make or buy plan had given us a first panorama of which could be our future industrial partners and in which areas we needed to find companies interested in the production of the components or in the execution of some of the processes. Involvement of the industry from the earliest moment for the most critical materials/components is vital. A dedicated web site was created [6] to present the future needs in a very technical and industry oriented format. Access to key technical reports and tendering documents is very important. In our case, there was also a major effort to involve the national contact points from the funding agencies as they have a better knowledge of their national industry. An early information system was establish so that they receive the information of future tenders several weeks/months before the starting of the procurement process to ensure that they had the time to motivate themselves their industry.

\subsubsection{Sourcing}

On the top of the previously mentioned actions, the project started already in 2012 several industrial sourcing campaigns. Four global industrial gathering were organized with global presentations but also with sessions dedicated to discuss directly with the WP Engineers and between industries to create consortiums and to identify subcontractors. In the last industrial event, more than one thousand meetings took place. The purpose of this type of events was to trigger discussions not on pure procurement but also on industrialization processes and early entry of the industry in the production. The global campaigns were complemented with national campaigns and with presentations in specialized technological fairs. The target was to avoid single supplier tenders and to rise the interest of the companies in working in innovative R\&D procurement where the knowledge transfer is one of the most valuable assets. 


\subsubsection{Tools to ensure the follow-up}

Tools are of paramount importance when we are procuring and producing components for a project of this size and duration. We should be able to operate the HL-LHC during several decades after their commissioning. What happened during production is not only important while producing but also during all the lifetime of the accelerator as nearly all components will have in a certain moment to be maintained or repaired. To ensure the production follow-up and its future operation, the project imposed common tools for the documentation and records handling. Those tools trace the components from their design to their manufacturing steps and any non-conformity, or test result can be retrieved in a simple and long term way. The tools are common independently of the origin of the supply, industry, in-kind or internal production. HL-LHC first Quality Plan was prepared in 2013.

\section{Present status and Lessons leant}

The project is now at its half way and for nearly all WPs the Development stage is nearly finished. If we consider the procurement of components, not considering the technical infrastructures, more than $50 \%$ of them have been already tendered or are today part of an in-kind contribution. Today we can start analysing if our strategy was adequate.

When the first consolidation budget was done in 2015, we had an average cost uncertainty of $[-30 \%-+50 \%]$. In this moment the cost deviation oscillates around the $[-5 \%-+5 \%]$ that means that we have managed to be on the target budget and on the lower part of the cost uncertainty. Nearly all the work axis have been fundamental for this success. The clear definition of the limit of the WPs and the strong review policy has avoided unnecessary R\&D and focused the research teams. The make or buy process allowed to find which components had to be done at a lab before going to industry. It also identified where we had not a large number of companies able to produce the components and in those case gave the tempo to launch early Market surveys. The sourcing campaigns were key to identify new suppliers so to avoid single suppliers tenders. The new infrastructures are today in use to make the industry produce on site instead of on their own premises reducing the cost of tooling and permitting and easy transfer of expertise. The traceability tools enable to prepare quickly the production folders and to transfer easily in house development to the industry.

We can conclude that the actions we took mitigated most part of the risks we had in front of us. Still, we had to face more than $20 \%$ of the tenders with a single supplier and we had not a perfect Gaussian price deviation curve. Under cost was strongly driven by pre prototyping and by increasing the number of tenderers. In that sense, the QUACO project [7] that was launched to demonstrate that industry could start working from the conceptual phase gives a beautiful example on how pre-commercial procurement is a perfect instrument to prepare industry to build new large research infrastructures. Including the industry from the conceptual phase industrialization time and cost can be reduced and the single supplier trap disappears.

\section{Conclusions}

Innovative research projects have to face several challenges that span from the difficulty to create a governance structure to find the right industrial partners to build their components. There are no magical ingredients for success but several of them that can help building resilience to adverse events. We can list as key ingredients: a clear governance and formal engagement of 
resources by all the partners, a strong PBS and TDR, schedule and cost baselines, the use of configuration, integration, documentation and records tracking tools, an early pragmatic make or buy analysis, an early involvement of industry with strong sourcing campaigns, the capacity to insource production of low TRL components and last but not least an intensive review policy in all the stages of the project.

\section{References}

[1] CERN, CERN/SPC/1068-CERN/FC/6014-CERN/3255, 2016

[2] HL-LHC Main www site - http://hilumilhc.web.cern.ch/about/collaboration-institutes.

[3] High-Luminosity Large Hadron Collider (HL-LHC) Technical Design Report DOI: http://dx.doi.org/10.23731/CYRM-2017-004

[4] I. Bejar Alonso, HL-LHC Quality Plan, https://edms.cern.ch/document/1513591, 2018

[5] ISO 16290:2013 Space systems --Definition of the Technology Readiness Levels (TRLs) and their criteria of assessment

[6] Industry Relations and Procurement Website for the HL-LHC project http://project-hl-1hcindustry.web.cern.ch/

[7] QUACO project, https://quaco.web.cern.ch/ European Union, under EU COFUND-PCP grant n. 689359 